\title{
Effect of external magnetic field on wakefield generation in underdense plasma slab using backward semi-Lagrangian Vlasov code
}

\author{
S. N. Razavinia and M. Ghorbanalilu* \\ Department of physics, Shahid Beheshti University, G. C., Tehran 1983969411, Iran
}

(Received 13 August 2019; published 25 November 2019; corrected 22 April 2020)

\begin{abstract}
Laser wakefield excitation in the interaction of femtosecond laser pulse with an underdense thin magnetized plasma slab is studied by one dimensional relativistic Vlasov-Maxwell system of equations. The interaction is modeled by relativistic Vlasov equation in propagation direction of laser pulse and fluid equations in transverse direction. Backward semi-Lagrangian method (BSL) is used for numerical solution of Vlasov equation. Generation of wakefields by a right and left handed circularly polarized (RCP) and (LCP) laser pulses in the interaction with nonmagnetized and magnetized plasma slab are examined and compared. Two directions are considered for external magnetic field, parallel and antiparallel to direction of laser pulse propagation. The results show that applying magnetic field along (opposite to) the propagation direction of RCP (LCP) laser pulse increases amplitude of density steepening and consequently wakefields amplitude, and mean kinetic energy of electrons compared with nonmagnetized plasma. The results are in complete agreement with previous analytical and numerical reports.
\end{abstract}

DOI: 10.1103/PhysRevAccelBeams.22.111305

\section{INTRODUCTION}

Because of breakdown limitation in conventional radio frequency accelerators, using alternative methods for charged particle acceleration is of great importance. Due to lack of electrical breakdown limitation in ionized plasma, plasma based accelerators are appropriate candidate for the next generation of particle accelerators [1-4]. These accelerators can generate electric fields more than $100 \mathrm{Gv} / \mathrm{m}$, which are three orders of magnitude larger than available electric fields in conventional accelerators, while acceleration length is reduced in the same order. These accelerators can accelerate electron beams to Gev energies in centimeter length [5-10]. The main concept of plasma based accelearators is using strong electric fields, which are generated in electrostatic plasma waves, for charged particle acceleration [11]. There are various methods for exciting large amplitude electrostatic waves in plasma such as: plasma wakefield acceleration (PWFA) [12-14], laser wakefield acceleration (LWFA) [15-19], and plasma beatwave acceleration (PBWA) [20].

Among the above mechanisms for exciting large amplitude plasma waves, laser wakefield acceleration is the most promising acceleration concept. In laser wakefield

\footnotetext{
"m_alilu@sbu.ac.ir
}

Published by the American Physical Society under the terms of the Creative Commons Attribution 4.0 International license. Further distribution of this work must maintain attribution to the author(s) and the published article's title, journal citation, and DOI. acceleration, by propagation of an ultra intense and ultra short laser pulse through underdense plasma, ponderomotive force of laser pulse pushes electrons of plasma forward, however ions due to their higher mass remain at rest. Charge separation between electrons and ions generates large amplitude electrostatic fields, named wakefield, behind laser pulse. The maximum amplitude of electrostatic field is achievable when laser pulse length is on the order of plasma wavelength. When laser intensity is high enough, a bubble or blow-out regime occurs. In this regime, ponderomotive force of laser pulse, in addition to longitudinal push of electrons also expels electrons transversely. Therefore, a periodically-repeated bubblelike structure (electron density inside the bubble is very low while at the sheath of bubble is very high) is created behind the laser pulse.

Electron bunches can either be injected into generated plasma wave, for instance by optical injection [21], ionization injection [22], and density-gradient injection [23,24] or detached from target plasma (self-injection), and by gaining energy from plasma wave accelerate to high energies. In self injection mechanism laser intensity is so high and plasma wave becomes highly nonlinear, therefore due to wave breaking $[25,26]$ some of the background electrons can be trapped and accelerated [27] in a plasma wave.

External and self-generated magnetic fields have significant effect on propagation [28] and self-focusing [29$31]$ of laser pulse, harmonic generation $[32,33]$, wakefield excitation, electron acceleration [34] etc.

In the presence of external magnetic field the generated wakefield becomes partially transverse and electromagnetic. For instance, the excited mode in the case of 
transverse magnetic field becomes an extraordinary mode with an electromagnetic part [35]. It is shown that an elliptically polarized laser pulse can excite upper-hybrid electrostatic wakefields in a transversely magnetized plasma [36]. Furthermore, in the interaction of a circularly polarized laser pulse with a longitudinally magnetized plasma, transverse wakefields do not arise and only longitudinal electrostatic wakefields are generated [37].

Optimization and controlling charge, energy, and quality of accelerated beam is one of the most critical issues in laser wakefield acceleration. Transverse and longitudinal external magnetic fields modify the structure of wakefields and could be used as a controlling knob for electron injection in laser wakefield acceleration. It has been shown that applying constant external magnetic field in the propagation direction of a right hand circularly polarized (RCP) laser pulse increases amplitude of the generated wakefields [37,38]. Moreover experimental [39] and twodimensional simulation [40-42] investigations demonstrate that, applying longitudinal external magnetic field of a few tens of tesla significantly enhances particle trapping in the bubble regime of laser wakefield acceleration (due to the suppression of the transverse drift of electrons), which cause to increase of the total charge and maximum energy of the trapped beam. However, the increase of trapped charge is at the cost of low control on energy spread and quality of accelerated beam.

If an external injection of electrons is used for electron beam generation, such as optical injection or density downramp injection, self-injection can have a negative effect on the quality of accelerated electron beam. Self-injection of electrons into laser wakefield can be controlled and suppressed by applying a longitudinal external magnetic field, which is approved by three-dimensional simulations for density down-ramp injection and also for optical injection in the bubble regime $[43,44]$.

On the other hand, applying transverse external magnetic filed of a few hundreds of tesla leads to the reduction of injection threshold and controllable self-injection of electrons in laser wakefield acceleration $[45,46]$. Furthermore, transverse magnetic field of a few tens of tesla could be used for controlling ionization injection in laser wakefield acceleration [47].

The Vlasov-Maxwell system of equations is one of the most widely used sets of equations for modeling relativistic laser plasma interaction. There are different methods for the numerical solution of the Vlasov equation which are categorized as: particle in cell (PIC) methods, Eulerian, and semi-Lagrangian Vlasov solvers. In the PIC method, plasma is approximated by finite number of superparticles and the Vlasov equation is integrated in time by advancing super particles along characteristic curves of the equation, while electromagnetic field equations are solved on grid $[48,49]$. PIC codes are powerful tools for studying dynamics of laser plasma interaction, even in two or three dimensions and complex geometries. Nevertheless, due to the introduction of superparticles rather than real particles in numerical simulation, they suffer from numerical noise which causes poor statistical resolution of particles motion. If the desired physics is in the low density region of phase space, where acceleration and trapping happened there, numerical noise becomes important. On the other hand, phase space grid based methods such as Eulerian [50,51] and semi-Lagrangian [52-55] Vlasov solvers, which directly solve the Vlasov equation as a partial differential equation in phase space, are free of numerical noise and give detailed structure of low density regions of phase space. The drawback of these methods is their requirement of a larger amount of computation resources compared with PIC methods.

In this paper we use one dimensional relativistic VlasovMaxwell system of equations for studying wakefield generation in the interaction of mildly relativistic circularly polarized laser pulse with a plasma slab in the presence of external longitudinal magnetic field. Wakefield excitation in magnetized plasma has already been studied by PIC [37,41] and Eulerian [56] codes. We use the backward semi-Lagrangian (BSL) method for studying excitation of wakefields in magnetized plasma slab. BSL code solves full kinetic one dimensional relativistic Vlasov-Maxwell equations along the magnetic field direction, and uses fluid equations in the direction perpendicular to the external magnetic field.

The validity of the 1D model is as long as the laser spot size $r_{s}$ is large compared to the plasma wavelength $\left(r_{s} \gg \lambda_{p}\right)$. Our simulation shows that magnetic field has an important role to electron wakefield acceleration. This role changes by direction of magnetic field and laser field polarization. For a RCP laser the parallel magnetic field enhances the electron wakefield acceleration, while for a LCP laser the antiparallel orientation plays a constructive role.

This paper is organized as following fashion. The system of equations which are relevant to interaction of laser pulse with magnetized plasma introduced in Sec. II. A brief review of BSL code algorithm presented in Sec. III. Wakefield structure, electron density fluctuations, time evolution of phase space, electrostatic energy of wakefield, and mean kinetic energy of electrons are investigated in the interaction of right and left-hand circularly polarized (LCP) laser pulses with a magnetized thin plasma slab. zThe magnetic field is oriented parallel and antiparallel to propagation direction of laser pulse. The results and comparison are given in Sec. IV. Finally, the conclusion is presented in Sec. V.

\section{THE RELEVANT EQUATIONS OF THE BSL CODE}

Ion motion is negligible during the interaction of high intensity short laser pulse with plasma, thus we consider 
ions as an immobile neutralizing background. We restrict ourselves to the one-dimensional model, which correspond to the broad laser beam assumption. Therefore, all quantities in the model depend only on the $x$ coordinate, which is the propagation direction of laser pulse. All of the equations and quantities in this paper are in normalized units. Time $t$ is normalized to inverse electron plasma frequency $\omega_{\mathrm{pe}}^{-1}$, space coordinate $x$ is normalized to $c \omega_{\mathrm{pe}}^{-1}$, momentum is normalized to $m_{e} c$, and finally electric and magnetic fields are normalized to $m_{e} c \omega_{\mathrm{pe}} / e$ and $m_{e} \omega_{\mathrm{pe}} / e$, respectively. Coulomb gauge $\nabla . \mathbf{A}=0$ is used for vector potential, which in one dimension indicates that vector potential is purely transverse $\mathbf{A}=\mathbf{A}_{\perp}(x, t)$. Plasma is embedded in an external magnetic field $\mathbf{B}_{0}=\sigma B_{0} \hat{\mathbf{x}}$, which depend on $\sigma= \pm 1$ is directed parallel or antiparallel to the laser pulse propagation direction. In our normalized units electron cyclotron frequency is defined as $\omega_{c}=-B_{0}$. Distribution function of electrons $F\left(x, p_{x}, \mathbf{p}_{\perp}, t\right)$ depends on four phase space variables, which require lots of computational effort for a full kinetic simulation. In order to reduce four dimensional to a two dimensional phase space we consider an initial situation in which all particles at the same coordinate $x$ have the same transverse momentum. Therefore, distribution function can be illustrated as $F\left(x, p_{x}, \mathbf{p}_{\perp}, t\right)=\delta\left(\mathbf{p}_{\perp}-\mathbf{P}_{\perp}(x, t)\right) f\left(x, p_{x}, t\right)$, where $f\left(x, p_{x}, t\right)$ describes electron motion along propagation direction of laser pulse and satisfies the relativistic Vlasov equation:

$$
\begin{aligned}
\frac{\partial f}{\partial t} & +\frac{p_{x}}{\gamma} \frac{\partial f}{\partial x}+\left(E_{x}(x, t)\right. \\
& \left.+\left(\mathbf{P}_{\perp}(x, t) \times \mathbf{B}_{\perp}(x, t)\right)_{x}\right) \frac{\partial f}{\partial p_{x}}=0,
\end{aligned}
$$

where $\gamma=\sqrt{1+p_{x}^{2}}$ is lorentz factor. $E_{x}(x, t)$ is electrostatic field and $\mathbf{B}_{\perp}(x, t)$ is the magnetic field of laser pulse. The longitudinal electric field $E_{x}$ can be obtained by poisson [Eq. (2a)] or Ampere [Eq. (2b)] equations.

$$
\begin{gathered}
\frac{\partial E_{x}(x, t)}{\partial x}=\rho(x, t)=n_{e}(x, t)-n_{0}, \\
\frac{\partial E_{x}(x, t)}{\partial t}=-J_{x}(x, t),
\end{gathered}
$$

where $n_{e}(x, t)$ is electron density, $n_{0}$ is initial electron density, which are equal to ion density before the entrance of laser pulse, and $J_{x}(x, t)$ is longitudinal current density which is defined as follow:

$$
\begin{gathered}
n_{e}(x, t)=\int_{-\infty}^{\infty} f\left(x, p_{x}, t\right) d p_{x}, \\
J_{x}(x, t)=\int_{-\infty}^{\infty} \frac{p_{x}}{\gamma} f\left(x, p_{x}, t\right) d p_{x} .
\end{gathered}
$$

We assume that transverse velocity is not relativistic and is calculated from fluid equations, while longitudinal velocity along laser propagation is relativistic and kinetic features of plasma preserved in this direction (longitudinal motion obey the relativistic Vlasov equation). Transverse momentum $\mathbf{P}_{\perp}$ is obtained from the introduction of transverse canonical momentum $\boldsymbol{\Pi}_{c \perp}(x, t)=\mathbf{P}_{\perp}(x, t)+\mathbf{A}_{\perp}(x, t)$ which satisfy following fluid equation:

$$
\begin{aligned}
& \left(\frac{\partial}{\partial t}+J_{x}(x, t) \frac{\partial}{\partial x}\right) \boldsymbol{\Pi}_{c \perp}(x, t) \\
& \quad=\boldsymbol{\Pi}_{c \perp}(x, t) \times \boldsymbol{\Omega}_{\mathbf{c}}-\mathbf{A}_{\perp}(x, t) \times \mathbf{\Omega}_{\mathbf{c}},
\end{aligned}
$$

where $\boldsymbol{\Omega}_{\mathbf{c}}=\sigma \omega_{c} \hat{\mathbf{x}}$. The above mentioned equations have already been presented for beat wave current drive [57] and raman forward scattering [58] in magnetized plasma. In the absence of external magnetic field $\boldsymbol{\Omega}_{\mathbf{c}}=0$, Eq. (4) is reduced to $d \boldsymbol{\Pi}_{c \perp}(x, t) / d t=0$, therefore $\boldsymbol{\Pi}_{c \perp}(x, t)$ is constant and without loss of generality we can assume that $\boldsymbol{\Pi}_{c \perp}(x, t)=0$, hence transverse momentum is obtained by $\mathbf{P}_{\perp}(x, t)=-\mathbf{A}_{\perp}(x, t)$.

Transverse electromagnetic fields for circularly polarized wave are computed from Maxwell equations.

$$
\begin{gathered}
\nabla \times \mathbf{E}_{\perp}(x, t)+\frac{\partial \mathbf{B}_{\perp}(x, t)}{\partial t}=0, \\
\nabla \times \mathbf{B}_{\perp}(x, t)=\mathbf{J}_{\perp}(x, t)+\frac{\partial \mathbf{E}_{\perp}(x, t)}{\partial t},
\end{gathered}
$$

where $\mathbf{J}_{\perp}$ is transverse current density:

$$
\mathbf{J}_{\perp}(x, t)=\mathbf{P}_{\perp}(x, t) n_{e}(x, t),
$$

provided that transverse electric field is known, vector potential can be calculated using the following equation:

$$
\mathbf{E}_{\perp}(x, t)=-\frac{\partial \mathbf{A}_{\perp}(x, t)}{\partial t} .
$$

In one dimensional problem transverse electromagnetic fields can be separated into left-going and right-going parts, $\mathbf{E}_{\perp}=\mathbf{E}_{l}+\mathbf{E}_{r}$ and $\mathbf{B}_{\perp}=\mathbf{B}_{l}+\mathbf{B}_{r}$. The left and rightgoing electric fields are defined as follows:

$$
\begin{aligned}
\mathbf{E}_{l} & =\frac{1}{2}\left(0, E_{y}-B_{z}, E_{z}+B_{y}\right), \\
\mathbf{E}_{r} & =\frac{1}{2}\left(0, E_{y}+B_{z}, E_{z}-B_{y}\right) .
\end{aligned}
$$

We define $E^{ \pm}=E_{y} \pm B_{z}$ and $F^{ \pm}=E_{z} \pm B_{y}$. By considering the wave number as $\mathbf{k}=k \hat{\mathbf{x}}$, all the left-going electromagnetic fields are eliminated $\left(E^{-}=F^{+}=0\right)$ and only the right-going fields would remain. Using this definition Maxwell equations can be rewritten as follow: 


$$
\begin{aligned}
& \left(\frac{\partial}{\partial t}+\frac{\partial}{\partial x}\right) E^{+}=-J_{y} . \\
& \left(\frac{\partial}{\partial t}-\frac{\partial}{\partial x}\right) F^{-}=-J_{z} .
\end{aligned}
$$

Equations (1) and (4) together with Eqs. (7) and (9) for transverse electromagnetic fields and Eq. (2) for electrostatic field, form the basic equations of BSL code.

\section{NUMERICAL SCHEME ALGORITHM}

Semi-Lagrangian method try to take advantage of both, Lagrangian and Eulerian points of view. In this scheme the Vlasov equation is integrated along its characteristics (Lagrangian point of view), then the distribution function is calculated on a Eulerian grid by a tensor product of B-splines. Characteristics of Vlasov equation are the solutions of the following system of equations:

$$
\begin{gathered}
\frac{d x}{d t}=\frac{p_{x}}{\gamma}, \\
\frac{d p_{x}}{d t}=E_{x}+\left(\mathbf{P}_{\perp} \times \mathbf{B}_{\perp}\right)_{x} .
\end{gathered}
$$

We define $X(t)=\left(x(t), p_{x}(t)\right)$ as the solution of characteristics and $U(X(t), t)=\left(p_{x} / \gamma, E_{x}+\left(\mathbf{P}_{\perp} \times \mathbf{B}_{\perp}\right)_{x}\right)$ as the force term. Then, we have:

$$
\begin{aligned}
\frac{d}{d t}[f(X(t), t)] & =\frac{\partial f}{\partial t}+\frac{d X}{d t} \cdot \nabla_{X} f \\
& =\frac{\partial f}{\partial t}+U[X(t), t] \cdot \nabla_{X} f=0 .
\end{aligned}
$$

Equation (11) indicates that the distribution function is constant along the characteristics of the Vlasov equation. The main idea of the semi-Lagrangian method is to use this feature for solving the Vlasov equation. In the BSL method, the values of the distribution function at phase space nodes updated by following characteristics, which ended at phase space grid points $\mathbf{x}=\left(x_{i}, p_{x j}\right)$ (where $i=$ $1 \ldots N_{x}, j=\ldots N_{p}$ are number of grid points in space and momentum space respectively), backward in time from $t_{n}+\Delta t$ to $t_{n}$ in order to find the starting point of characteristics. Then, the value of the distribution function at $t_{n}+\Delta t$ is computed by cubic B-spline interpolation from the value of the distribution function at the starting point of characteristics, provided that the value of the distribution function is known at phase space nodes in $t_{n}$. Therefore, the BSL algorithm has two main steps: 1. Finding the starting point of Vlasov characteristics ending at $\mathbf{x}$, i.e., $X\left(t_{n}\right) .2$. Calculate $f\left(X\left(t_{n}\right), t_{n}\right)$ by cubic B-spline interpolation, provided that $f\left(\mathbf{x}, t_{n}\right)$ is known, and using constancy of distribution function along characteristics $f\left(\mathbf{x}, t_{n}+\Delta t\right)=f\left(X\left(t_{n}\right), t_{n}\right)$.
In order to perform the first step, Eqs. (10a) and (10b) should be solved. The force term at the right-hand side of Eq. (10b) is obtained by solving Eq. (2a) for $E_{x}$, Eqs. (9a) and (9b) are integrated along their vacuum characteristics $x=-t$ for $E^{+}$and $F^{-}\left(E_{y}=B_{z}=E^{+} / 2, E_{z}=-B_{y}=F^{-} / 2\right)$ and Eq. (7) for $A_{y}$ and $A_{z}$ (using $E_{y}$ and $E_{z}$ ). Equation (4) is solved by the Crank-Nicholson method for $\boldsymbol{\Pi}_{c \perp}$, then transverse momentum $\mathbf{P}_{\perp}$ is computed from the relation, $\mathbf{P}_{\perp}=\boldsymbol{\Pi}_{c \perp}-\mathbf{A}_{\perp}$.

Detailed description of the semi-Lagrangian method and cubic spline interpolation can be found in Refs. [52,59].

\section{WAKEFIELD EXCITATION IN A MAGNETIZED THIN PLASMA SLAB}

We consider an underdense homogeneous plasma slab with $n_{0}=0.01 n_{c}$, which is surrounded by vacuum and transition (in which plasma density rise from zero to $n_{0}$ ) regions at both sides. Length of plasma layer, vacuum region and transition region are $L_{\text {plasma }}=40 c \omega_{\text {pe }}^{-1}$, $L_{\text {vacuum }}=1.2 c \omega_{\text {pe }}^{-1}, L_{\text {jump }}=1.8 c \omega_{\text {pe }}^{-1}$, respectively. Initial distribution function is assumed Maxwellian and temperature of electrons is considered $3 \mathrm{keV}$. Phase space is sampled by $N_{x} N_{p x}=1300 \times 400$ grid points. Plasma is embedded in external constant magnetic field strength of 1200 Tesla. Electron cyclotron frequency is defined as $\omega_{c}=e B_{0} / m_{e}$, therefore by using the dimensionless relation $\omega_{c} / \omega_{\mathrm{pe}}=B_{0}$ (gauss) $/ 3.21 \times 10^{-3} \sqrt{n_{e}\left(\mathrm{~cm}^{-3}\right)} \quad$ we obtain $\omega_{c}=1.12 \omega_{\mathrm{pe}}$. Direction of $\boldsymbol{\Omega}_{\mathbf{c}}$ is determined by the direction of externally applied magnetic field $\left(\boldsymbol{\Omega}_{\mathbf{c}}=\sigma \omega_{c} \hat{\mathbf{x}}\right)$. For instance, if the external magnetic field is in the positive (negative) $x$ direction then $\Omega_{c}=1.12 \omega_{\mathrm{pe}}$ $\left(\Omega_{c}=-1.12 \omega_{\mathrm{pe}}\right)$. Production of such intense magnetic field is reported in Ref. [60].

Circularly polarized laser pulse with half-sinusoidal envelope enters the system from vacuum at left boundary and propagates in plasma toward the right boundary. Forward propagating RCP and LCP laser pulses introduce as follows:

$$
\begin{aligned}
& E_{\mathrm{RCP}}^{+}=2 E_{0} \sin \left(\frac{\pi \xi}{L}\right) \cos \left(k_{0} x-\omega_{0} t\right), \\
& F_{\mathrm{RCP}}^{-}=-2 E_{0} \sin \left(\frac{\pi \xi}{L}\right) \sin \left(k_{0} x-\omega_{0} t\right), \\
& E_{\mathrm{LCP}}^{+}=2 E_{0} \sin \left(\frac{\pi \xi}{L}\right) \cos \left(k_{0} x-\omega_{0} t\right), \\
& F_{\mathrm{LCP}}^{-}=2 E_{0} \sin \left(\frac{\pi \xi}{L}\right) \sin \left(k_{0} x-\omega_{0} t\right),
\end{aligned}
$$

where $k_{0}$ and $\omega_{0}=10 \omega_{\mathrm{pe}}$ are wave number and frequency of the laser pulse, $L=\lambda_{p}=2 \pi c \omega_{\mathrm{pe}}^{-1}$ is length of the laser pulse envelope ( $\lambda_{p}$ is plasma wavelength), $\xi=x-t$ and 


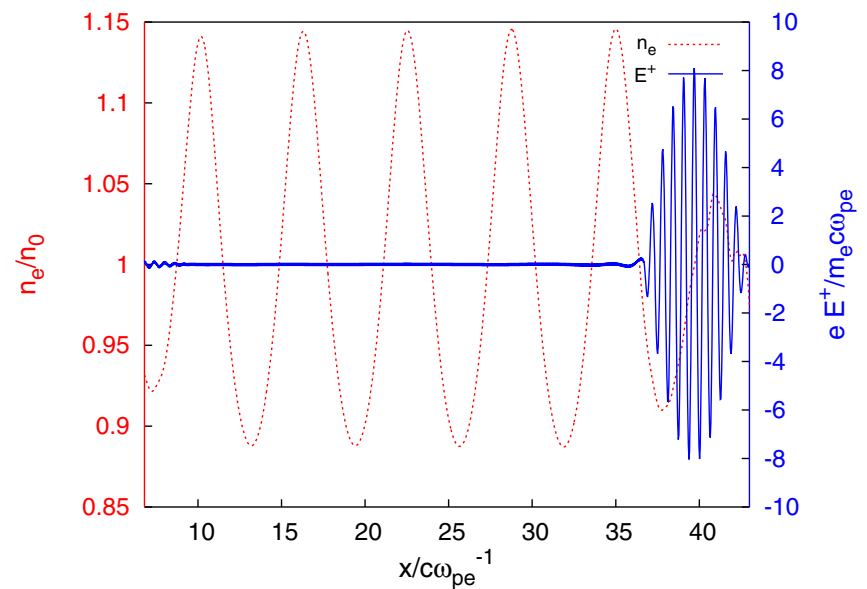

(a)

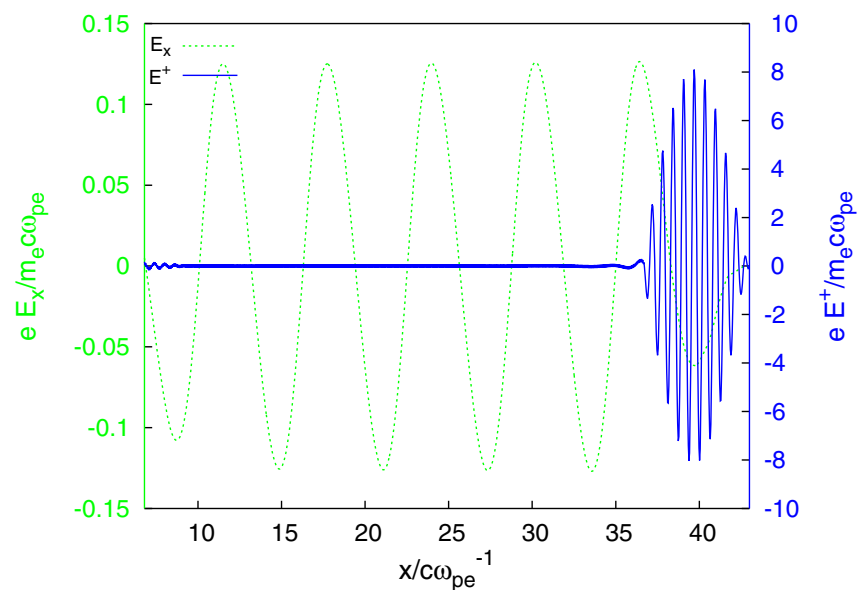

(b)

FIG. 1. (a) Electron density fluctuations $n_{e}$ (red dot line) and subsequently (b) electrostatic wakefields $E_{x}$ (green dot line) which are induced in plasma during the propagation of RCP laser pulse (which its electric field $E^{+}$is displayed by solid blue line) in a nonmagnetized underdense plasma slab. Figures are plotted at $t / \omega_{\mathrm{pe}}^{-1} \simeq 42$ after the start of simulation.

$E_{0}=a_{0} \omega_{0}$ is the amplitude of laser pulse. Only in the interval $-L<\xi<0$ and $L_{\text {vacuum }}+L_{\text {jump }}<t<L+L_{\text {vacuum }}+$ $L_{\text {jump }}, E_{0}$ in nonzero. Wavelength of the laser is equal to $\lambda_{0}=1 \mu \mathrm{m}$ and $a_{0}=0.4$ is normalized amplitude of laser vector potential, which corresponds to a laser intensity with $I_{0}=4.3 \times 10^{17} \mathrm{~W} / \mathrm{cm}^{2}$.

The results of the BSL code for wakefields generated by RCP laser pulse in nonmagnetized plasma are presented in Fig. 1. By propagation of laser pulse through plasma, ponderomotive force of laser pulse $F_{p} \sim \nabla a^{2}$ pushes electrons forward, whereas ions due to their larger mass cannot respond to ponderomotive force and remain at rest. Charge separation between electrons and ions generate a longitudinal electron wave (plasma wave) that follows the laser pulse. Periodic density peaks and electronfree regions are created behind the laser pulse, which are evident in Fig. 1(a). The front and back of the laser pulse kicks electrons forward and backward, therefore the distance between density peaks is approximately equal to plasma wavelength (because $L=\lambda_{p}$ ). As a result of charge separation, large amplitude electrostatic fields $E_{x}$ (wakefields) are generated in plasma, which are displayed in Fig. 1(b). The maximum amplitude of electrostatic field $E_{x} \sim 0.14$, is in agreement with theoretical value of maximum electric field generated by a plane polarized pulse in cold plasma: $E_{\max }=a_{0}^{2} / \sqrt{1+a_{0}^{2}}\left(m_{e} c \omega_{\mathrm{pe}} / e\right)$, where $m_{e} c \omega_{\mathrm{pe}} / e$ is the wavebreaking limit in cold plasma [18,19].

The aforementioned value for the wavebreaking limit is based on cold fluid theory. The warm wavebreaking field is less than the cold one. Therefore, including thermal effects can significantly reduce the maximum plasma wave amplitude relative to the cold wavebreaking value [61]. Opposition of plasma pressure to the tendency of plasma density to increase to infinity together with the thermal velocity of particles moving in the direction of the wave, which enables them to be trapped at a lower wave amplitude than if they were initially stationary, cause the reduction of the maximum plasma wave amplitude. In the present simulation, temperature of electrons is considered $3 \mathrm{keV}$, therefore, thermal velocity of electrons $\left(v_{\text {thermal }}=0.07 c\right)$ is much less than the quiver velocity of an electron $\left(v_{\text {quiver }}=0.37 c\right)$ in the laser field and we can neglect the thermal effects. It should be noted that considering thermal effects will reduce the maximum plasma wave amplitude compared with the value which we reported here.

In order to investigate the role of external magnetic field we consider parallel $(\sigma=1)$ and antiparallel $(\sigma=-1)$ directions for magnetic field at this stage. Density peaks created by propagation of RCP (LCP) laser pulse in nonmagnetized $(\sigma=0)$ plasma, plasma with parallel $(\sigma=1)$ and anti-parallel $(\sigma=-1)$ magnetic fields, are compared in Fig. 2(a) (Fig. 2(b)). The figures show that for RCP laser pulse the density peaks amplitude is enhanced for parallel and weakened for antiparallel directions, while for LCP laser pulse the scenario is reversed. Consequently, amplitude of wakefield is enhanced in the former and suppressed in the latter structure which is evident in Fig. 3(a) [Fig. 3(b)]. Our results are in agreement with analytical and numerical works, that have been done in Refs. [37,38].

According to the previous analytical investigation [38], the ratio of wakefield potential in a magnetized plasma $\left(\phi_{\mathrm{em}}\right)$ to a nonmagnetized plasma $\left(\phi_{0}\right)$ is as follow:

$\frac{\phi_{\mathrm{em}}}{\phi_{0}} \approx \eta_{e}=\frac{c^{2}}{v_{\mathrm{ge}}^{2}}\left[1 \pm\left(1-\frac{X}{1 \mp Y}\right)^{\frac{1}{2}} \frac{Y}{1 \mp Y} \frac{v_{\mathrm{ge}}}{c}\right] \frac{1}{1 \mp Y}$

Where, $\quad X=\omega_{\mathrm{pe}}^{2} / \omega^{2}, \quad Y=\sigma \omega_{c} / \omega, \quad$ and $\quad v_{\mathrm{ge}} / c=$ $[1-X /(1 \mp Y)]^{1 / 2} /\left[1 \pm X Y / 2(1 \mp Y)^{2}\right]$. Here, we consider the $\sigma$ as $\mp$ signs (the upper sign is for RCP laser pulse and lower sign is for LCP laser pulse). According to the 


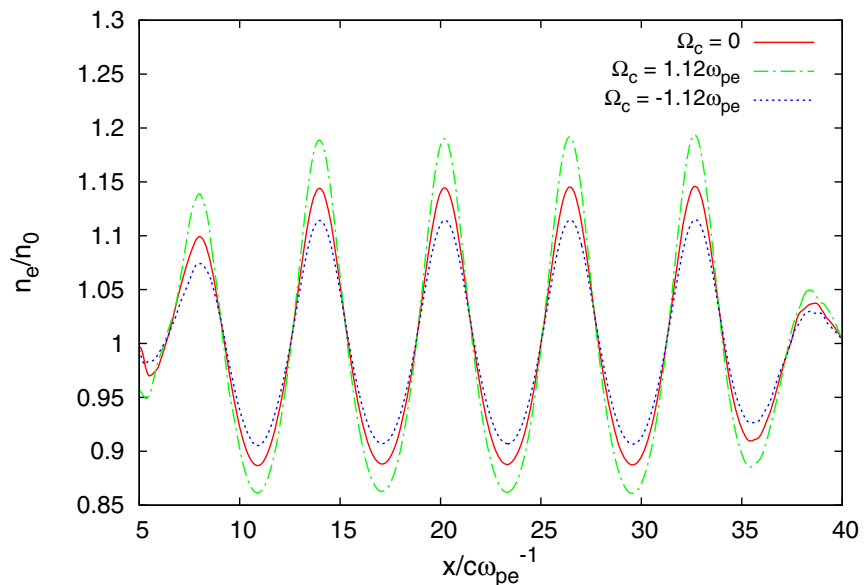

(a)

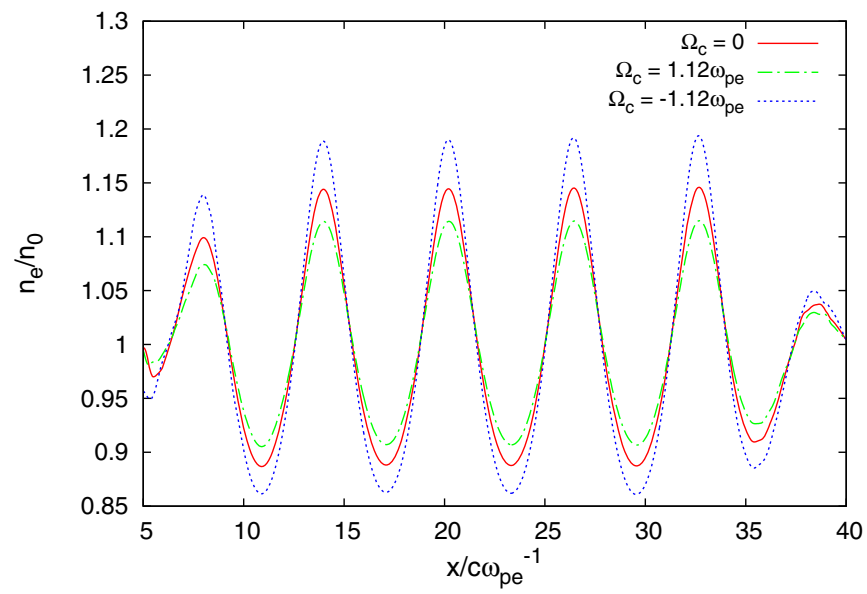

(b)

FIG. 2. Comparison of electron density fluctuations $n_{e}$ induced by propagation of (a) RCP and (b) LCP laser pulses in a nonmagnetized plasma $\Omega_{c}=0$ (solid red line), plasma with an external magnetic field in positive $x$ direction $\Omega_{c}=1.12 \omega_{\mathrm{pe}}$ (green dash dot line) and negative $x$ direction $\Omega_{c}=-1.12 \omega_{\mathrm{pe}}$ (blue dot line) at $t / \omega_{\mathrm{pe}}^{-1} \simeq 40$ after the start of simulation.

aforementioned relation [Eq. (14)], the antiparallel structure with a RCP laser pulse is the same with parallel LCP laser pulse structure. On the other hand, we find that the wakefield potential in magnetized plasma are intricately related to the magnetic field strength and direction, chirality of $C P$ laser pulse and its group velocity $v_{\text {ge }}$. Therefore, no simple physical description is possible. However, if $\eta_{e}$ is plotted against $Y$ for a fixed value of $X=10^{-4}$ (which is the same as the parameter we regard in our simulation where $\omega_{0}=10 \omega_{\mathrm{pe}}$ ), the results show that $\eta_{e}$ increase (decrease) for RCP (LCP) laser pulse with the increase of $\omega_{c}$ (Fig. 1 and Fig. 2 in Ref. [38]). In spite of this, the physical description is possible for the resonance condition when the laser pulse frequency closes to the electron cyclotron frequency, however, the resonance situation is beyond of our scope in this paper. When the resonance

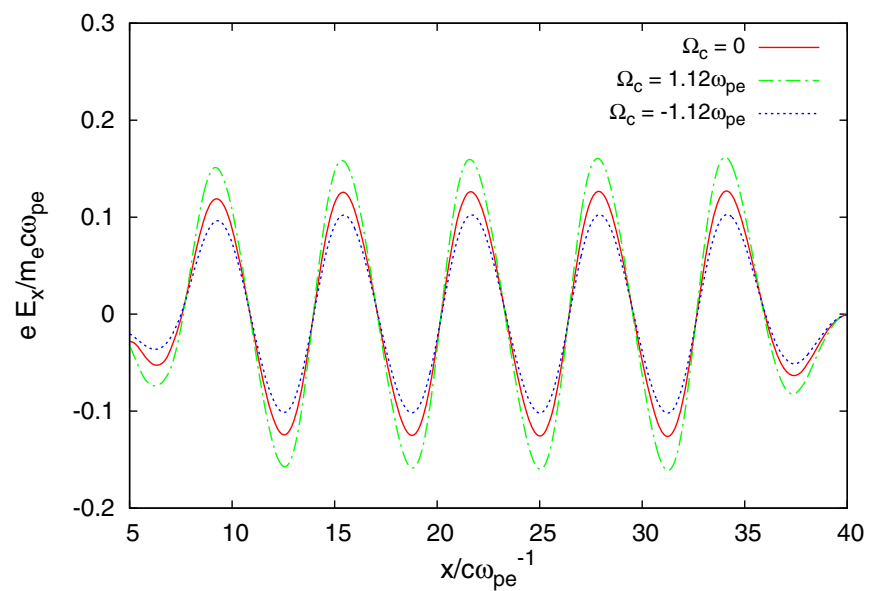

(a)

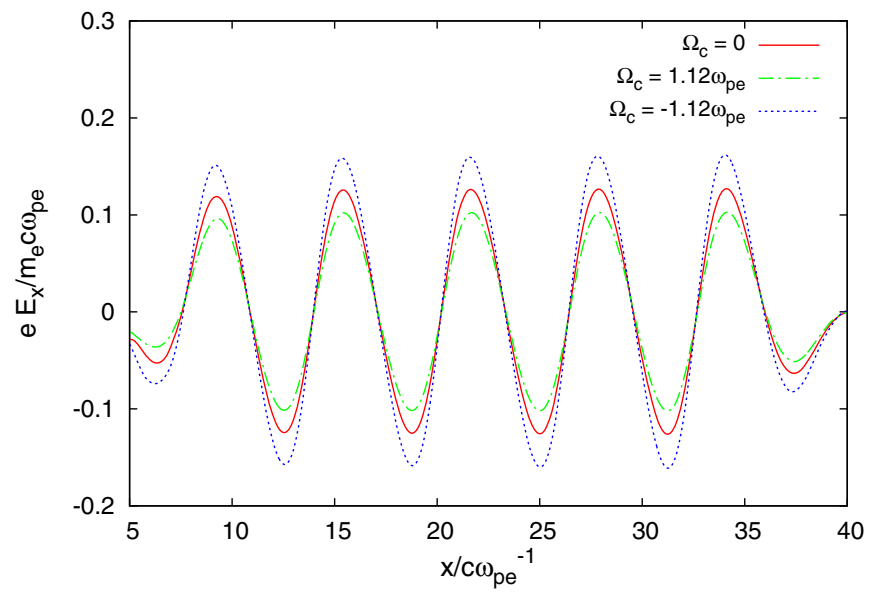

(b)

FIG. 3. Comparison of wakefields $E_{x}$ generated by propagation of (a) RCP and (b) LCP laser pulses in a nonmagnetized plasma $\Omega_{c}=0$ (solid red line), plasma which are embedded in an external magnetic field in positive $x$ direction $\Omega_{c}=1.12 \omega_{\mathrm{pe}}$ (green dash dot line) and negative $x$ direction $\Omega_{c}=-1.12 \omega_{\mathrm{pe}}$ (blue dot line) at $t / \omega_{\mathrm{pe}}^{-1} \simeq 40$ after the start of simulation.

condition is satisfied for parallel RCP structure, the electron and laser field rotate in the same direction with the same frequency and electron absorbed most part of the laser field energy resonantly.

Comparison of time evolution of normalized longitudinal $\left[\int\left\{\int\left[\gamma\left(x, p_{x}\right)-1\right] f\left(x, p_{x}\right) d p_{x}\right\} d x / L_{\text {system }}\right]$ and transverse $\left(\int\left(p_{y}^{2}(x, t)+p_{z}^{2}(x, t)\right) d x / L_{\text {system }}\right)$ mean kinetic energy of electrons during the interaction of RCP laser pulse with plasma, for $\boldsymbol{\Omega}_{\mathbf{c}}=0, \boldsymbol{\Omega}_{\mathbf{c}}=1.12 \omega_{\mathrm{pe}} \hat{\mathbf{x}}$ and $\boldsymbol{\Omega}_{\mathbf{c}}=$ $-1.12 \omega_{\mathrm{pe}} \hat{\mathbf{x}}$ has demonstrated in Figs. 4(a) and 4(b), respectively. Motion of electrons in transverse direction is considered nonrelativistic and obtained from fluid equations, while the longitudinal motion of electrons is relativistic and obtained from kinetic equations. Applying external magnetic field in the positive $x$ direction (parallel to longitudinal motion of electrons) causes increments of 


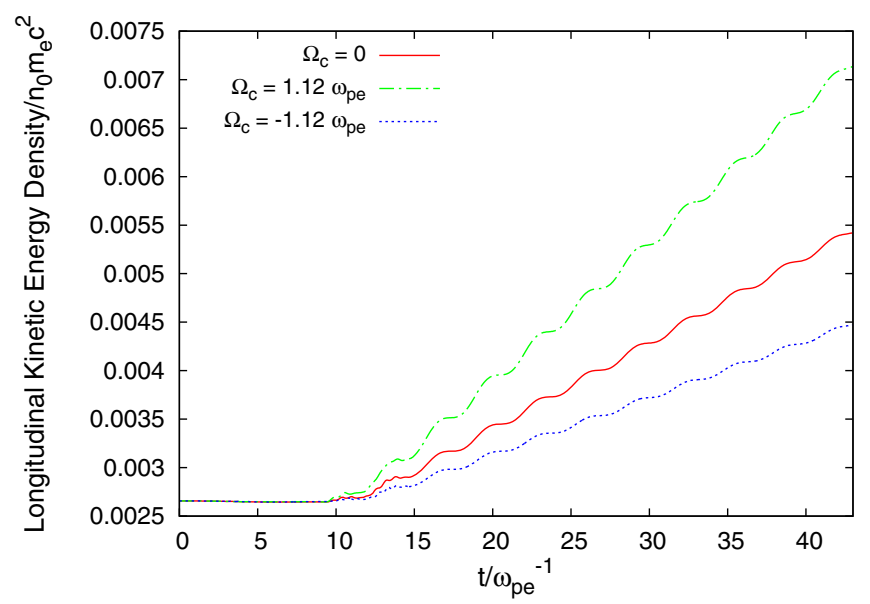

(a)

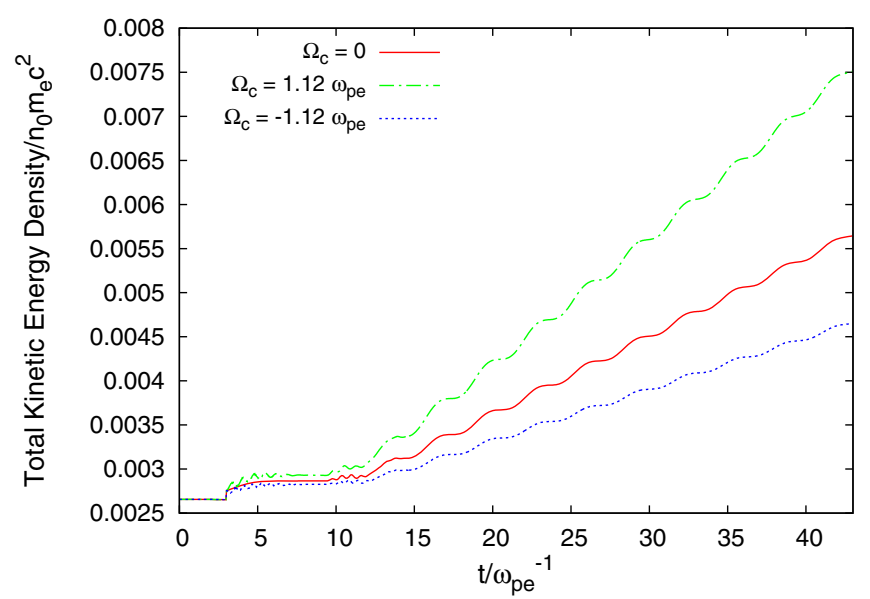

(c)

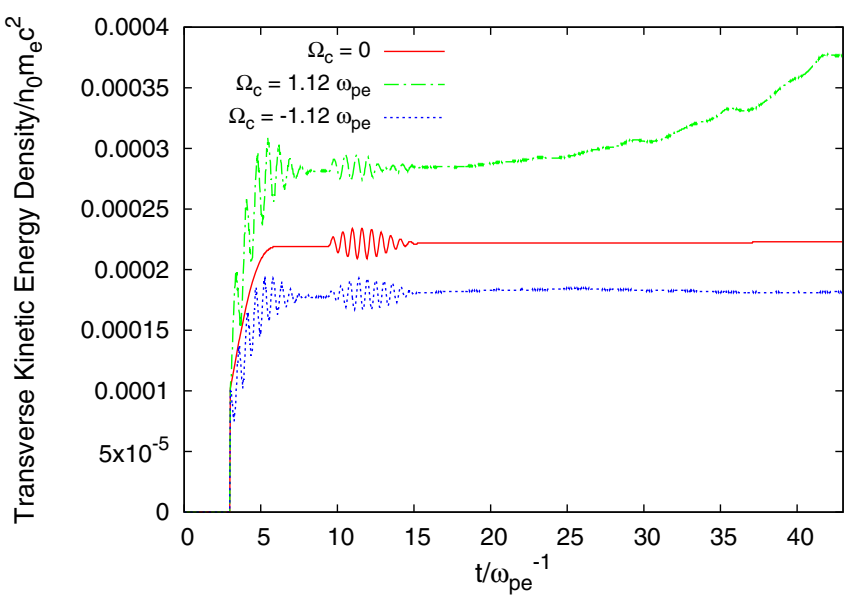

(b)

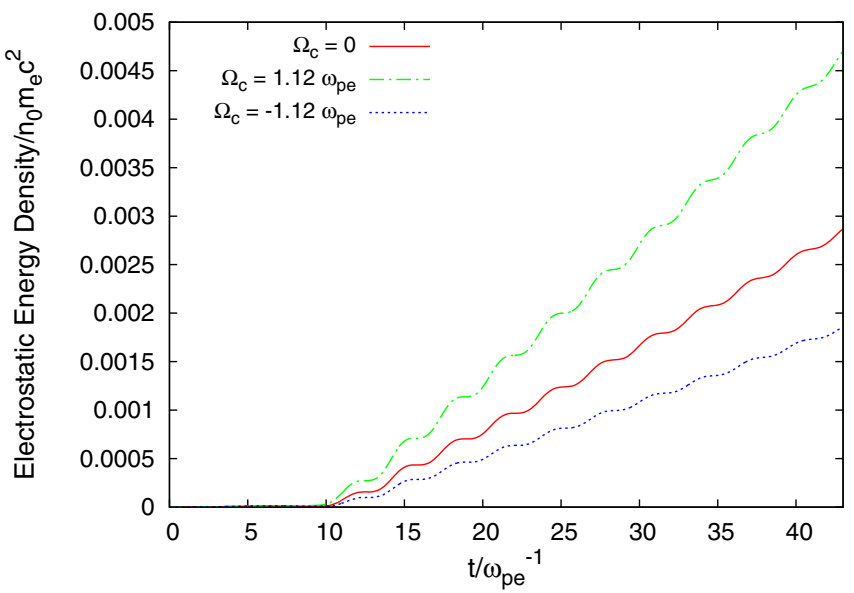

(d)

FIG. 4. Comparison of time evolution of (a) longitudinal mean kinetic energy density of electrons, (b) transverse mean kinetic energy density of electrons, (c) total kinetic energy density of electrons, and (d) electrostatic energy density of wakefields, which are generated by RCP laser pulse in a nonmagnetized plasma $\Omega_{c}=0$ (red solid line), plasma which are embedded in an external magnetic field in positive $x$ direction $\Omega_{c}=1.12 \omega_{\mathrm{pe}}$ (green dash dot line) and negative $x$ direction $\Omega_{c}=-1.12 \omega_{\mathrm{pe}}$ (blue dot line).

longitudinal and transverse momentum of electrons compared with that of without an external magnetic field. However, it has less influence on transverse motion of electrons compared with longitudinal motion. For instance, in this case the transverse kinetic energy of electrons increases $15.9 \%$, while longitudinal kinetic energy increases $31.6 \%$ compared with a nonmagnetized plasma. For the case of external magnetic field in negative $x$ direction, transverse and kinetic longitudinal energy of electrons decrease $18.8 \%$ and $17.7 \%$ because of the presence of the static magnetic field, respectively. Relativistic longitudinal motion of electrons constitute the main part of the total kinetic energy density of electrons which are evident in Fig. 4(c). Finally, total mean kinetic energy of electrons for a RCP laser with parallel structure increases $33.1 \%$ compared with a nonmagnetized plasma. However, this value for the antiparallel structure decreases $17.6 \%$.
The time evolution of wakefield (excited by RCP laser pulse) electrostatic energy $\left(\int E_{x}^{2} d x / 2 L_{\text {system }}\right)$ for $\boldsymbol{\Omega}_{\mathbf{c}}=0$, $\boldsymbol{\Omega}_{\mathbf{c}}=1.12 \omega_{\mathrm{pe}} \hat{\mathbf{x}}$ and $\boldsymbol{\Omega}_{\mathbf{c}}=-1.12 \omega_{\mathrm{pe}} \hat{\mathbf{x}}$ are presented in Fig. 4(d). This figure illustrates that by applying the magnetic field in positive $x$ direction, wakefield energy (at the end of simulation) increases $63.8 \%$, while for magnetic field in negative $x$ direction decreases $35.2 \%$ compared with the nonmagnetized case.

The time evolution of electron distribution function in the case of wakefield excitation in underdense plasma slab by RCP laser pulse is depicted in Fig. 5. It is evident from contour plots of the distribution function in Fig. 5(a) that before transmission of laser pulse through plasma, most of electrons are distributed around zero momentum and only a few of them have momentums approximately $\pm 0.5 m_{e} c$. Generation of stronger (weaker) wakefields by applying a magnetic field in positive (negative) $x$ direction compared with the nonmagnetized case is obvious in 


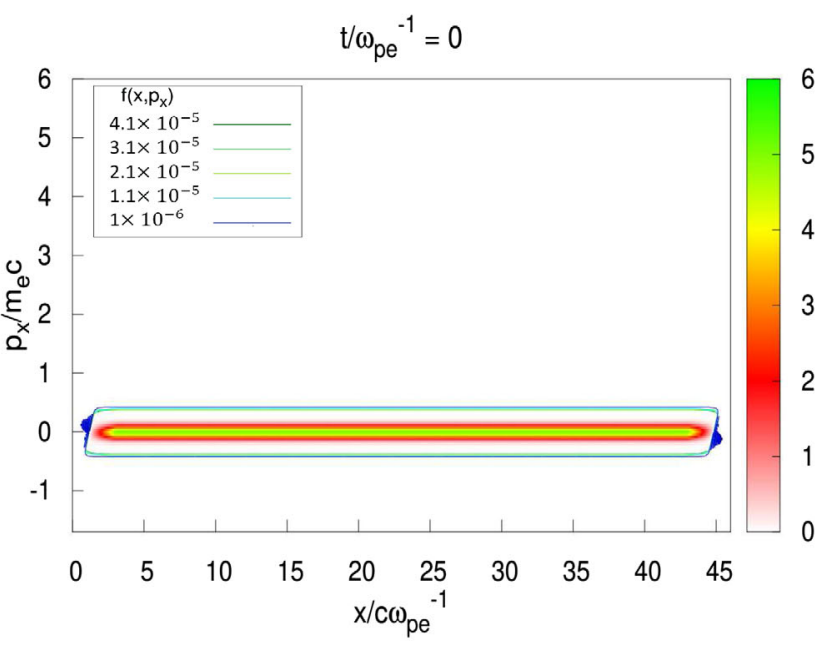

(a)

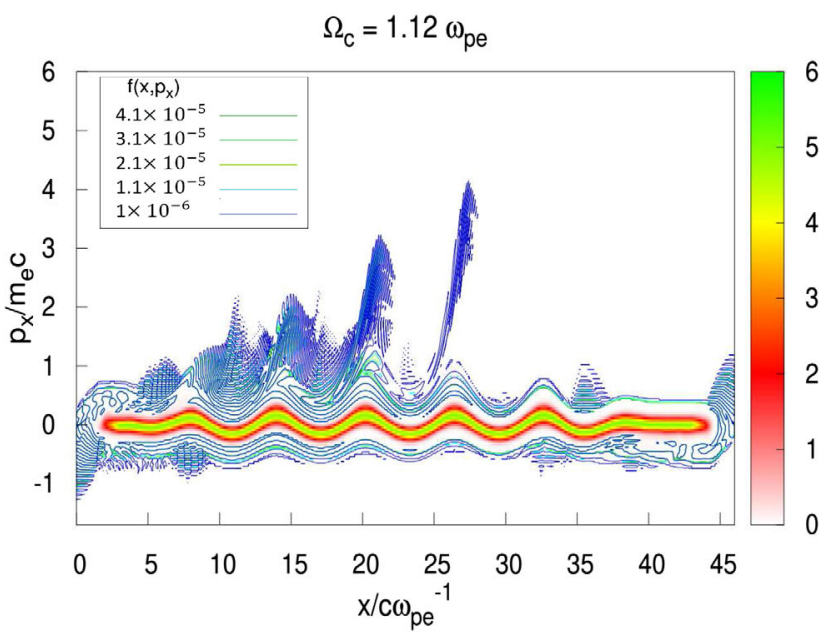

(c)

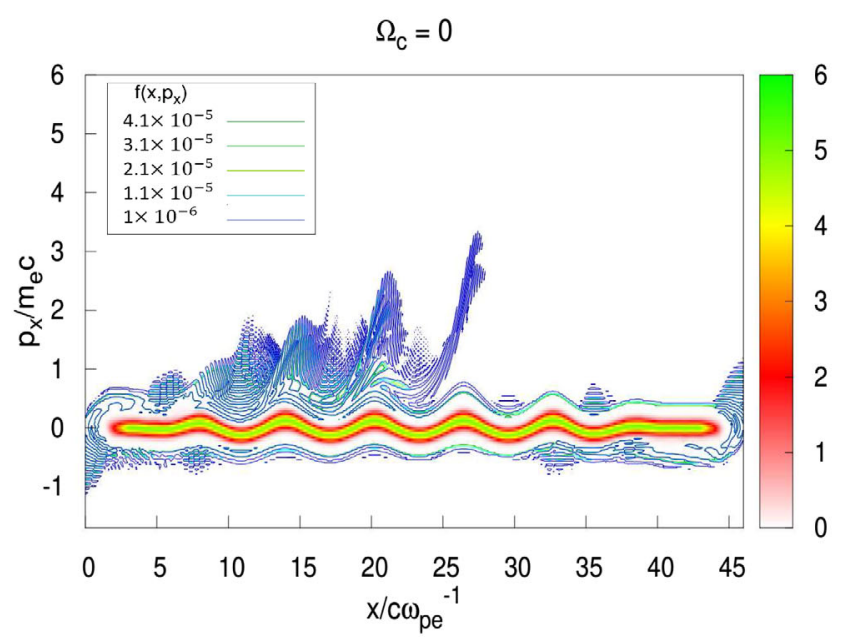

(b)

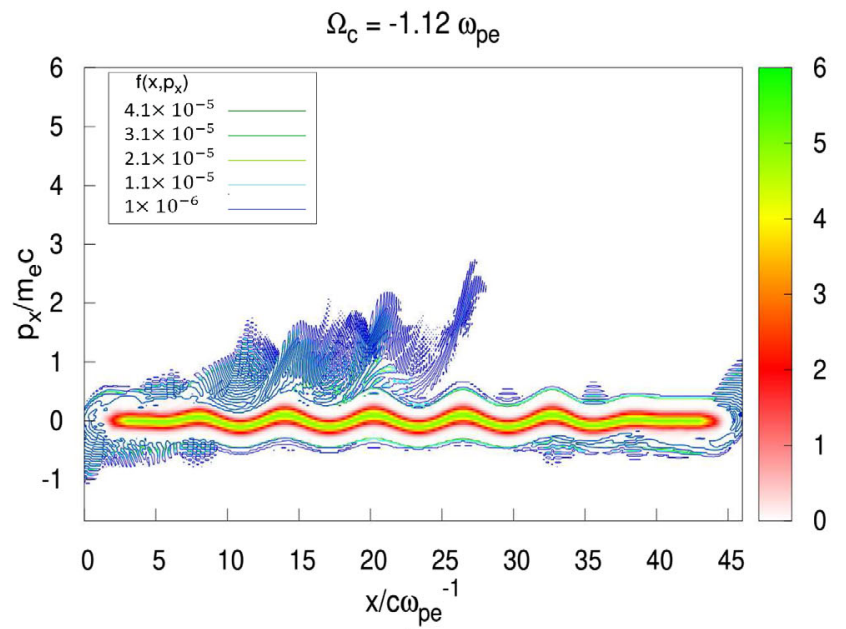

(d)

FIG. 5. Contour plots of electron distribution function $f\left(x, p_{x}\right)$ in phase space $\left(x, p_{x}\right)$ : (a) before the entrance of RCP laser pulse through plasma slab $\left(t / \omega_{\mathrm{pe}}^{-1}=0\right)$ and at the time $t / \omega_{\mathrm{pe}}^{-1} \simeq 40$ for (b) nonmagnetized plasma slab $\Omega_{c}=0$, (c) plasma slab which are embedded in an external magnetic field in positive $x$ direction $\Omega_{c}=1.12 \omega_{\mathrm{pe}}$ and (d) plasma slab which are embedded in an external magnetic field in negative $x$ direction $\Omega_{c}=-1.12 \omega_{\mathrm{pe}}$.

Figs. 5(c) and 5(d). Contour plots of electron distribution function $f\left(x, p_{x}\right)$ in phase space $\left(x, p_{x}\right)$ for a nonmagnetized plasma at $t / \omega_{\mathrm{pe}}^{-1} \simeq 40$ represented in Fig. 5(b). By propagation of laser pulse through plasma, a few fractions of electrons detach from plasma bulk and accelerate to momentums up to $3 m_{e} c$. However, by applying magnetic field in positive $x$ direction electrons can gain momentum up to $4 m_{e} c$ [Fig. 5(c)] in similar time, while this value for magnetic field in negative $x$ direction is up to $2.5 m_{e} c$ [Fig. 5(d)]. Distribution function of electrons and its sinusoidal shape are in accordance with density peaks in Fig. 2(a).

The electric field of a RCP laser pulse rotates in the same (opposite) direction as the electrons in a plasma which are embedded in an external parallel (antiparallel) magnetic field. As a result, transverse and longitudinal velocities of electrons increase (decrease) in the presence of magnetic field. Consequently, for parallel structure higher density peaks are created compared to a nonmagnetized plasma and the reverse is happened for antiparallel structure. As a consequence of higher (lower) density peaks formation, higher (lower) wakefields are generated and therefore, longitudinal momentum of electrons increase (decrease) in comparison to a nonmagnetized plasma. For example, Fig. 5(c) shows enhancement of electrons acceleration with respect to a nonmagnetized plasma slab in Fig. 5(b), while for antiparallel structure in Fig. 5(d) the electron acceleration is suppressed by external magnetic field compared to a nonmagnetized plasma slab.

The time evolution of electron density profile $n_{e}(x, t)$ (in $x-t$ space), in the case of wakefield excitation by RCP laser pulse interaction with a nonmagnetized plasma slab 


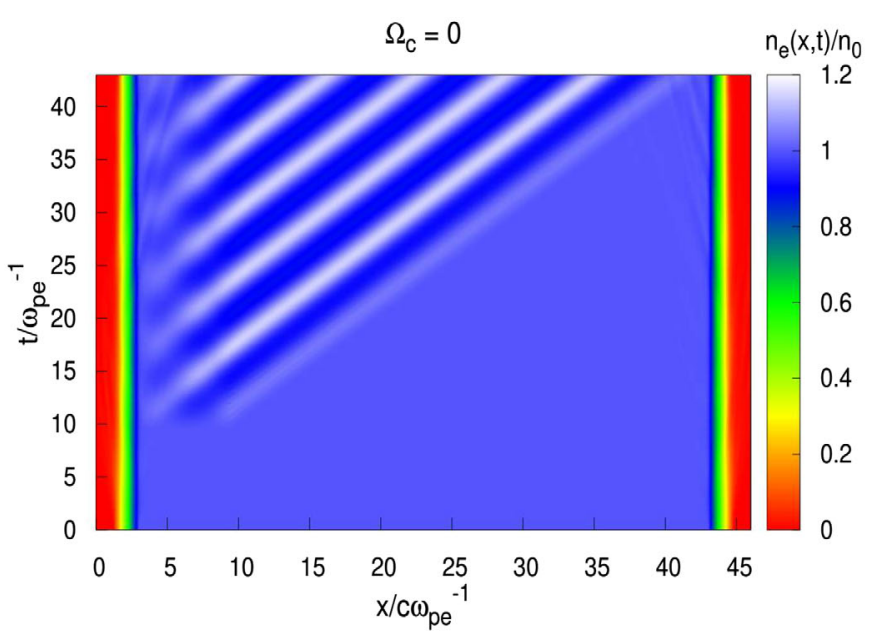

(a)

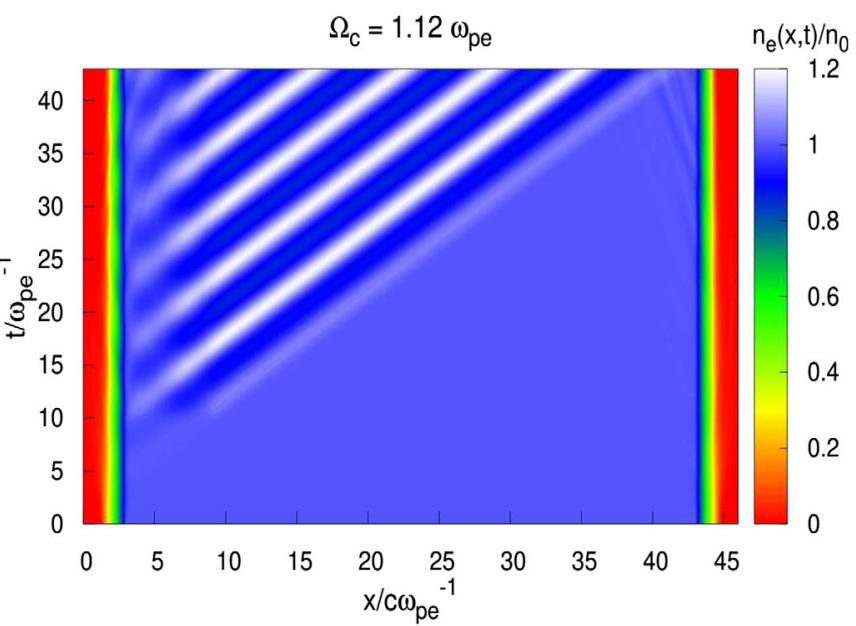

(b)

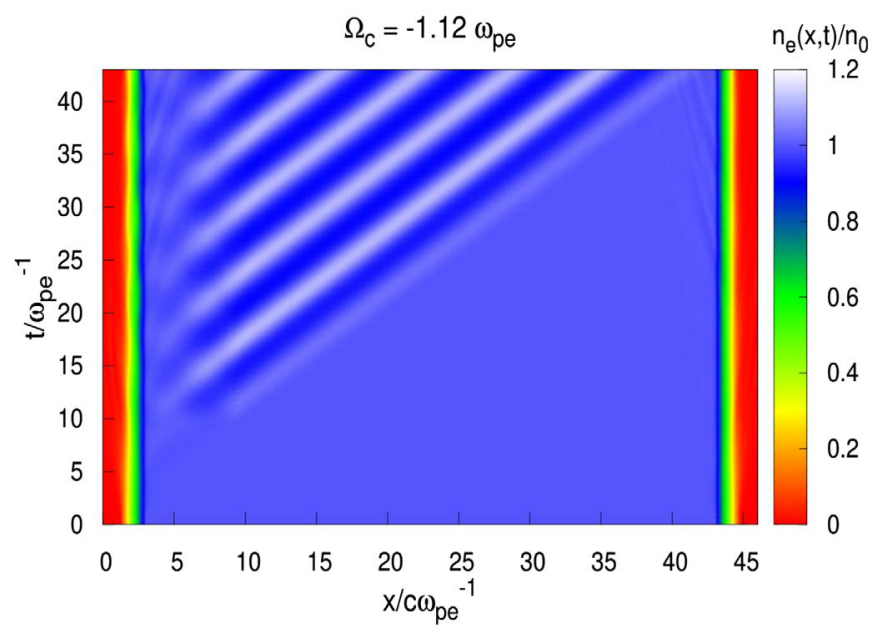

(c)

FIG. 6. Time evolution of electron plasma density profile $n_{e}(x, t)$ in the $(x, t)$ space. Plasma density fluctuations are created by propagation of RCP laser pulse through (a) nonmagnetized plasma slab $\Omega_{c}=0$, (b) plasma slab which are embedded in an external magnetic field in positive $x$ direction $\Omega_{c}=1.12 \omega_{\mathrm{pe}}$ and (c) plasma slab which are embedded in an external magnetic field in negative $x$ direction $\Omega_{c}=-1.12 \omega_{\mathrm{pe}}$.

$\boldsymbol{\Omega}_{\mathbf{c}}=0$ [Fig. 6(a)], plasma slab embedded in an external magnetic field in positive $x$ direction $\boldsymbol{\Omega}_{\mathbf{c}}=1.12 \omega_{\mathrm{pe}} \hat{\mathbf{x}}$ [Fig. 6(b)], and negative $x$ direction $\boldsymbol{\Omega}_{\mathbf{c}}=-1.12 \omega_{\mathrm{pe}} \hat{\mathbf{x}}$ [Fig. 6(c)] are presented in Fig. 6. According to Fig. 6(a), for times greater than $t / \omega_{\mathrm{pe}}^{-1} \simeq 10$ wakefields are generated in plasma slab and before this time there is no the wakefield in plasma slab. Red and green regions on both sides of this figure display vacuum (length of this region is equal to $L_{\text {vacuum }}$ ) and transition (jump region which its length is introduced by $L_{\text {jump }}$ ) regions, which are considered on both side of plasma slab. The electron density varies periodically by moving along the $x$-axis for a given time. The same situation occurred for moving along the time axis for a fixed point $x$ inside slab. Comparison of different panels of Fig. 6 show that by applying magnetic field in positive $x$ direction [Fig. 6(b)] sharper density peaks are generated in magnetized plasma slab compared with a nonmagnetized case, while the situation is opposite for the case of applied magnetic field in negative $x$ direction and density peaks become weakened [Fig. 6(c)].

In Fig. 7, time evolution of longitudinal part of normalized kinetic energy density $\left(\int\left(\gamma\left(x, p_{x}\right)-1\right) f\left(x, p_{x}\right) d p_{x}\right)$ of electrons (in $x-t$ space), for wakefield excitation by RCP laser pulse in a nonmagnetized plasma slab $\boldsymbol{\Omega}_{\mathbf{c}}=0$ [Fig. 7(a)], plasma slab embedded in an external magnetic field in positive $x$ direction $\boldsymbol{\Omega}_{\mathbf{c}}=1.12 \omega_{\mathrm{pe}} \hat{\mathbf{x}}$ [Fig. 7(b)], and in negative $x$ direction $\boldsymbol{\Omega}_{\mathbf{c}}=-1.12 \omega_{\mathrm{pe}} \hat{\mathbf{x}}$ [Fig. 7(c)] are displayed. As indicated in Fig. 4(a), 4(b), longitudinal momentum dominates the kinetic energy of electrons. Peaks of longitudinal kinetic energy correspond to electron density peaks in Fig. 6.

Applying a magnetic field in the positive $x$ direction causes the increment of the kinetic energy density of electrons in the plasma slab. As it is evident in Fig. 7(b) 


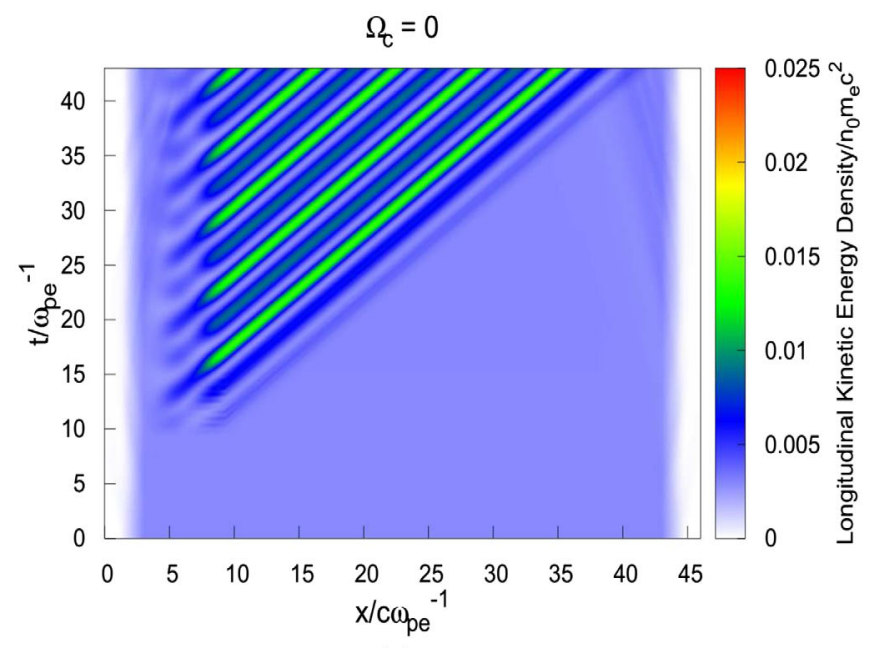

(a)

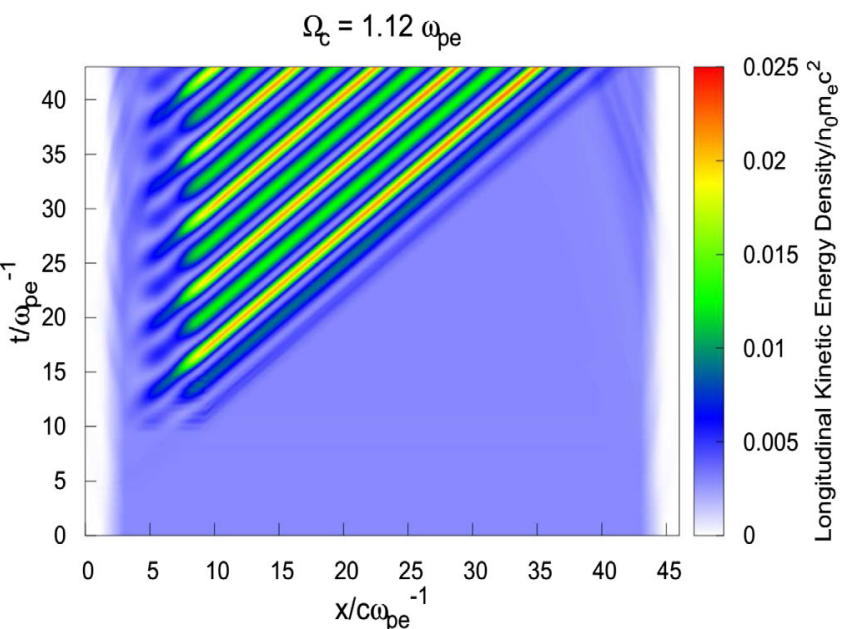

(b)

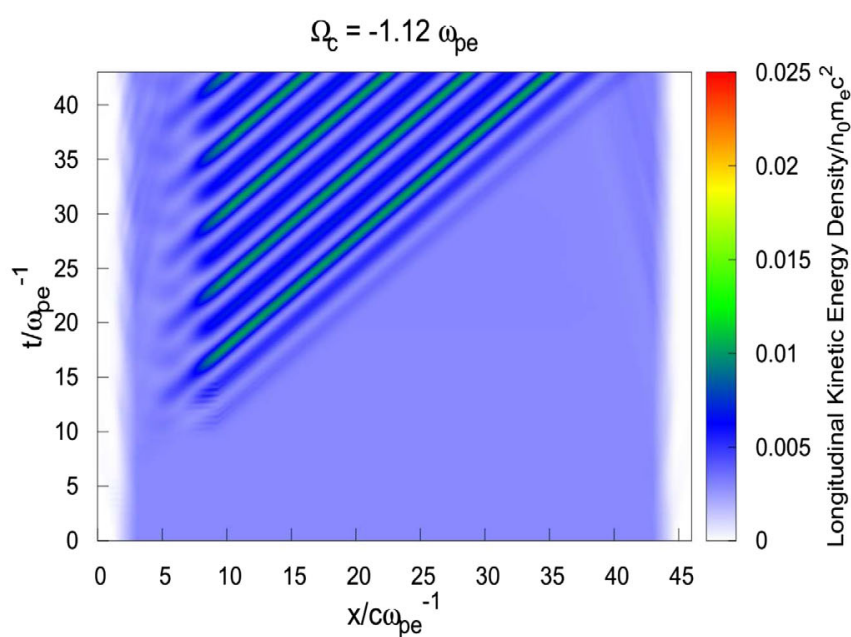

(c)

FIG. 7. Time evolution of normalized longitudinal kinetic energy density profile in the $(x, t)$ space, which are related to the propagation of RCP laser pulse through (a) nonmagnetized plasma slab $\Omega_{c}=0$, (b) plasma slab which are embedded in an external magnetic field in positive $x$ direction $\Omega_{c}=1.12 \omega_{\mathrm{pe}}$ and (c) plasma slab which are embedded in an external magnetic field in negative $x$ direction $\Omega_{c}=-1.12 \omega_{\mathrm{pe}}$.

peaks of kinetic energy are sharper and have larger values than those of the nonmagnetized plasma slab in Fig. 7(a). While, for a plasma slab with antiparallel structure, electrons cannot reach to the maximum value of kinetic energy [Fig. 7(c)] and maximum value of their peaks are lower than that of nonmagnetized plasma slab.

The influence of external longitudinal magnetic field on external injection of electrons in laser wakefield has already been investigated in the three-dimensional bubble regime (for linearly polarized laser pulse) $[43,44]$. It is shown that an external longitudinal magnetic field causes the sheath electrons in the bubble regime to obtain an azimuthal velocity (time varying magnetic flux induces an azimuthal electric field) and rotate reversely around the laser axis. Therefore, a huge longitudinal magnetic field is generated in plasma which strengthens the initial longitudinal magnetic field strongly. The magnetic field causes the bubble rear to open up and sheath electrons cannot reach the laser axis, due to strong centrifugal force. These effects cannot be revealed in a two-dimensional planar symmetry configuration due to the vanishing of the centrifugal force [62]. Therefore, electric charge at the rear of the bubble on axis is reduced and leads to the reduction of longitudinal electric field, which is responsible for electron trapping. Consequently, self-injection can be suppressed. Thereby, the accelerated electron beam in external injection is not altered by self-injection.

Three-dimensional simulations of the effect of transverse magnetic field on wakefield excitation by linearly polarized laser pulse show that wakefield structure altered significantly and off-axis asymmetric injection of electrons into the wakefield occurred $[45,46]$. On the other hand, the transverse magnetic field relaxes the longitudinal trapping condition and modifies transverse trapping condition [47]. 


\section{DISCUSSION AND CONCLUSION}

We have studied wakefield excitation by propagation of a circularly polarized laser pulse through a thin plasma slab using the Vlasov-Maxwell system of equations. It is found that applying an external magnetic field in the proper direction can increase the amplitude of wakefield. In order to investigate the effect of the external magnetic field on wakefield generation, we have used nonrelativistic fluid equations in the perpendicular direction and the relativistic Vlasov equation in the parallel direction of the laser pulse. Fluid equations are solved by the Crank-Nickolson method, while the Vlasov equation is solved by backward semiLagrangian method. The BSL method provides detailed description of distribution function of electrons in phase space, even in very low density regions. Moreover it gives information about average behavior of the system and, due to fine resolution in phase space, is a powerful tool for detailed investigation of particle dynamics.

Our simulation revealed that in the interaction of a RCP laser pulse with a magnetized plasma slab, the parallel external magnetic field enhanced wakefield amplitude, increased electrostatic energy of wakefield and mean kinetic energy of electrons, while an antiparallel magnetic field attenuated wakefield, decreased electrostatic energy of wakefield and mean kinetic energy. The simulation indicated the reversed behaviour for LCP laser pulse. In summary, the presence of an external magnetic field along (opposite to) the propagation direction of RCP (LCP) laser pulse increased the amplitude of density peaks, electrostatic energy of wakefield, and mean kinetic energy of electrons.

[1] S. M. Hooker, Developments in laser-driven plasma accelerators, Nat. Photonics 7, 775 (2013).

[2] E. Esarey, C. B. Schroeder, and W. P. Leemans, Physics of laser-driven plasma-based electron accelerators, Rev. Mod. Phys. 81, 1229 (2009).

[3] V. Malka, J. Faure, Y. A. Gauduel, E. Lefebvre, A. Rousse, and K. T. Phuoc, Principles and applications of compact laser-plasma accelerators, Nat. Phys. 4, 447 (2008).

[4] R. Bingham, J. T. Mendonca, and P. K. Shukla, Plasma based charged-particle accelerators, Plasma Phys. Controlled Fusion 46, R1 (2004).

[5] X. Yang, E. Brunetti, D. R. Gil, G. H. Welsh, F. Y. Li, S. Cipiccia, B. Ersfeld, D. W. Grant, P. A. Grant, M. R. Islam, M. P. Tooley, G. Vieux, S. M. Wiggins, Z. M. Sheng, and D. A. Jaroszynski, Three electron beams from a laserplasma wakefield accelerator and the energy apportioning question, Sci. Rep. 7, 43910 (2017).

[6] W. P. Leemans, A. J. Gonsalves, H.-S. Mao, K. Nakamura, C. Benedetti, C. B. Schroeder, C. Tóth, J. Daniels, D. E. Mittelberger, S. S. Bulanov, J.-L. Vay, C. G. R. Geddes, and E. Esarey, Multi-GeV Electron Beams from CapillaryDischarge-Guided Subpetawatt Laser Pulses in the SelfTrapping Regime, Phys. Rev. Lett. 113, 245002 (2014).
[7] H. T. Kim, K. H. Pae, H. J. Cha, I. J. Kim, T. J. Yu, J. H. Sung, S. K. Lee, T. M. Jeong, and J. Lee, Enhancement of Electron Energy to the Multi-GeV Regime by a Dual-Stage Laser-Wakefield Accelerator Pumped by Petawatt Laser Pulses, Phys. Rev. Lett. 111, 165002 (2013).

[8] W. Lu, M. Tzoufras, C. Joshi, F. S. Tsung, W. B. Mori, J. Vieira, R. A. Fonseca, and L. O. Silva, Generating multi$\mathrm{GeV}$ electron bunches using single stage laser wakefield acceleration in a 3D nonlinear regime, Phys. Rev. Accel. Beams 10, 061301 (2007).

[9] W. P. Leemans, B. Nagler, A. J. Gonsalves, C. Tóth, K. Nakamura, C. G. R. Geddes, E. Esarey, C. B. Schroeder, and $\mathrm{S}$. Hooker, GeV electron beams from a centimetrescale accelerator, Nat. Phys. 2, 696 (2006).

[10] M. Kando et al., Electron acceleration by a nonlinear wakefield generated by ultrashort (23-fs) high-peak-power laser pulses in plasma, Phys. Rev. E 71, 015403 (2005).

[11] T. Tajima and J. M. Dawson, Laser Electron Accelerator, Phys. Rev. Lett. 43, 267 (1979).

[12] P. Chen, J. M. Dawson, R. W. Huff, and T. Katsouleas, Acceleration of Electrons by the Interaction of a Bunched Electron Beam with a Plasma, Phys. Rev. Lett. 54, 693 (1985).

[13] C. Joshi, Review of beam driven plasma wakefield accelerators, AIP Conf. Proc. 737, 3 (2004).

[14] C. Joshi, E. Adli, W. An, C. E. Clayton, S. Corde, S. Gessner, M. J. Hogan, M. Litos, W. Lu, and K. A. Marsh, Plasma wakefield acceleration experiments at FACET II, Plasma Phys. Controlled Fusion 60, 034001 (2018).

[15] S. V. Bulanov, T. Z. Esirkepov, Y. Hayashi, H. Kiriyama, J. K. Koga, H. Kotaki, M. Mori, and M. Kando, On some theoretical problems of laser wake-field accelerators, J. Plasma Phys. 82, 905820308 (2016).

[16] C. G. R. Geddes et al., Computational studies and optimization of wakefield accelerators, J. Phys. 125, 012002 (2008).

[17] V. I. Berezhiani and I. G. Murusidze, Relativistic wakefield generation by an intense laser pulse in a plasma, Phys. Lett. A 148, 338 (1990).

[18] A. Ting, E. Esarey, and P. Sprangle, Nonlinear wake-field generation and relativistic focusing of intense laser pulses in plasmas, Phys. Fluids B: Plasma Physics 2, 1390 (1990).

[19] P. Sprangle, E. Esarey, and A. Ting, Nonlinear interaction of intense laser pulses in plasmas, Phys. Rev. A 41, 4463 (1990).

[20] C. E. Clayton, K. A. Marsh, M. Everett, A. Lal, and C. Joshi, in Proceedings of International Conference on Particle Accelerators, Vol. 4 (IEEE, Washington, 1993), pp. 2551-2555.

[21] J. Faure, C. Rechatin, A. Norlin, A. Lifschitz, Y. Glinec, and V. Malka, Controlled injection and acceleration of electrons in plasma wakefields by colliding laser pulses, Nature (London) 444, 737 (2006).

[22] M. Chen, E. Esareya, C. B. Schroeder, C. G. R. Geddes, and W. P. Leemans, Theory of ionization-induced trapping in laser-plasma accelerators, Phys. Plasmas 19, 033101 (2012).

[23] C. G. R. Geddes, K. Nakamura, G. R. Plateau, Cs. Toth, E. Cormier-Michel, E. Esarey, C. B. Schroeder, J. R. Cary, 
and W. P. Leemans, Plasma-Density-Gradient Injection of Low Absolute-Momentum-Spread Electron Bunches, Phys. Rev. Lett. 100, 215004 (2008).

[24] M. Hansson, B. Aurand, X. Davoine, H. Ekerfelt, K. Svensson, A. Persson, C.-G. Wahlström, and O. Lundh, Down-ramp injection and independently controlled acceleration of electrons in a tailored laser wakefield accelerator, Phys. Rev. Accel. Beams 18, 071303 (2015).

[25] A. Modena, Z. Najmudin, A. E. Dangor, C. E. Clayton, K. A. Marsh, C. Joshi, V. Malka, C. B. Darrow, C. Danson, D. Neely, and F. N. Walsh, Electron acceleration from the breaking of relativistic plasma waves, Nature (London) 377, 606 (1995).

[26] S. V. Bulanov, F. Pegoraro, A. M. Pukhov, and A. S. Sakharov, Transverse-Wake Wave Breaking, Phys. Rev. Lett. 78, 4205 (1997).

[27] E. Esarey and M. Pilloff, Trapping and acceleration in nonlinear plasma waves, Phys. Plasmas 2, 1432 (1995).

[28] S. X. Luan, W. Yu, F. Y. Li, D. Wu, Z. M. Sheng, M. Y. Yu, and J. Zhang, Laser propagation in dense magnetized plasma, Phys. Rev. E 94, 053207 (2016).

[29] T. C. Wilson, F. Y. Li, M. Weikum, and Z. M. Sheng, Influence of strong magnetic fields on laser pulse propagation in underdense plasma, Plasma Phys. Controlled Fusion 59, 065002 (2017).

[30] M. Ghorbanalilu, Focusing of intense laser beam by a thin axially magnetized plasma lens, Phys. Plasmas 17, 023111 (2010).

[31] M. Ghorbanalilu and B. Shokri, Investigation of optical properties of an overdense magnetized plasma lens in the interaction with high-intensity Gaussian laser pulses, Appl. Phys. B 124, 38 (2018).

[32] P. Jha, R. K. Mishra, G. Raj, and A. K. Upadhyay, Second harmonic generation in laser magnetized-plasma interaction, Phys. Plasmas 14, 053107 (2007).

[33] M. Ghorbanalilu, Second and third harmonics generation in the interaction of strongly magnetized dense plasma with an intense laser beam, Laser Part. Beams 30, 291 (2012).

[34] X. Xia, B. Xu, and L. Wang, Research on electron acceleration in interaction of intense laser and magnetized plasma by ponderomotive force, Optik 127, 658 (2016).

[35] G. Brodin and J. Lundberg, Excitation of electromagnetic wake fields in a magnetized plasma, Phys. Rev. E 57, 7041 (1998).

[36] P. K. Shukla, Generation of wakefields by elliptically polarized laser pulses in a magnetized plasma, Phys. Plasmas 6, 1363 (1999).

[37] P. Jha, A. Saroch, R. K. Mishra, and A. K. Upadhyay, Laser wakefield acceleration in magnetized plasma, Phys. Rev. Accel. Beams 15, 081301 (2012).

[38] P. K. Shukla, Laser wakefield generation in magnetized plasmas, Phys. Scr. T52, 73 (1994).

[39] T. Hosokai, K. Kinoshita, A. Zhidkov, A. Maekawa, A. Yamazaki, and M. Uesaka, Effect of External Static Magnetic Field on the Emittance and Total Charge of Electron Beams Generated by Laser-Wakefield Acceleration, Phys. Rev. Lett. 97, 075004 (2006).
[40] M. Drouin, A. Bourdier, Q. Harry, and S. Rassou, Influence of a static magnetic field on beam emittance in laser wakefield acceleration, J. Mod. Phys. 3, 1991 (2012).

[41] M. S. Hur, D. N. Gupta, and H. Suk, Enhanced electron trapping by a static longitudinal magnetic field in laser wakefield acceleration, Phys. Lett. A 372, 2684 (2008).

[42] A. Bourdier, G. Girard, S. Rassou, X. Davoine, and M. Drouin, Influence of a magnetic guide field on selfinjection in wakefield acceleration, J. Mod. Phys. 3, 1983 (2012).

[43] Q. Zhao, S. M. Weng, M.Chen, M. Zeng, B. Hidding, D. A. Jaroszynski, R. Assmann, and Z. M. Sheng, Sub-femtosecond electron bunches in laser wakefield acceleration via injection suppression with a magnetic field, Plasma Phys. Controlled Fusion 61, 085015 (2019).

[44] S. Rassou, A. Bourdier, and M. Drouin, Influence of a strong longitudinal magnetic field on laser wakefield acceleration, Phys. Plasmas 22, 073104 (2015).

[45] J. Vieira, S. F. Martins, V. B. Pathak, R. A. Fonseca, W. B. Mori, and L. O. Silva, Magnetic Control of Particle Injection in Plasma Based Accelerators, Phys. Rev. Lett. 106, 225001 (2011).

[46] J. Vieira, J. L. Martins, V. B. Pathak, R. A. Fonseca, W. B. Mori, and L. O. Silva, Magnetically assisted selfinjection and radiation generation for plasma-based acceleration, Plasma Phys. Controlled Fusion 54, 124044 (2012).

[47] Q. Zhao, S. M. Weng, Z. M. Sheng, M.Chen, G. B. Zhang, W. B. Mori, B. Hidding, D. A. Jaroszynski, and J. Zhang, Ionization injection in a laser wakefield accelerator subject to a transverse magnetic field, New J. Phys. 20, 063031 (2018).

[48] C. K. Birdsall and A. B. Langdon, Plasma Physics Via Computer simulation (McGraw-Hill, Inc., New York, 1985).

[49] J. M. Dawson, Particle simulation of plasmas, Rev. Mod. Phys. 55, 403 (1983).

[50] M. Shoucri, Eulerian codes for the numerical solution of the Vlasov equation, Commun. Nonlinear Sci. Numer. Simul. 13, 174 (2008).

[51] D. J. Strozzi, M. M. Shoucri, and A. Bers, Study of laser plasma interactions using an Eulerian Vlasov code, Comput. Phys. Commun. 164, 156 (2004).

[52] E. Sonnendrücker, J. Roche, and P. B. A. Ghizzo, The Semi-Lagrangian Method for the Numerical Resolution of the Vlasov Equation, J. Comput. Phys. 149, 201 (1999).

[53] D. D. Sarto, A. Ghizzo, T. Reveille, N. Besse, and P. Bertrand, Application of a semi-Lagrangian scheme in the relativistic regime of laser interaction with an overdense plasma slab, Commun. Nonlinear Sci. Numer. Simul. 13, 59 (2008).

[54] M. L. Bégué, A. Ghizzo, P. Bertrand, E. Sonnendrücker, and O. Coulaud, Two-dimensional semi-Lagrangian Vlasov simulations of laser-plasma interaction in the relativistic regime, J. Plasma Phys. 62, 367 (1999).

[55] G. Lehmann, Efficient Semi-Lagrangian Vlasov-Maxwell simulations of high order harmonic generation from relativistic laser-plasma interactions, Commun. Comput. Phys. 20, 583 (2016). 
[56] M. Shoucri, Numerical simulation of Raman and Brillouin laser-pulse amplification in a magnetized plasma, Laser Part. Beams 34, 315 (2016).

[57] A. Ghizzo, P. Bertrand, M. Shoucri, T. Johnston, E. Fijalkow, M. Feix, and V. Demchenko, Study of laserplasma beat wave current drive with an Eulerian Vlasov code, Nucl. Fusion 32, 45 (1992).

[58] P. Bertrand, A. Ghizzo, S. J. Karttunen, T. J. H. Pättikangas, R. R. E. Salomaa, and M. Shoucri, Simulations of waveparticle interactions in stimulated Raman forward scattering in a magnetized plasma, Phys. Fluids B: Plasma Phys. 4, 3590 (1992).

[59] N. Crouseilles, G. Latu, and E. Sonnendrücker, A parallel Vlasov solver based on local cubic spline interpolation on patches, J. Comput. Phys. 228, 1429 (2009).
[60] D. Nakamura, A. Ikeda, H. Sawabe, Y. H. Matsuda, and S. Takeyama, Record indoor magnetic field of $1200 \mathrm{~T}$ generated by electromagnetic flux-compression, Rev. Sci. Instrum. 89, 095106 (2018).

[61] T. Katsouleas and W. B. Mori, Wave-Breaking Amplitude of Relativistic Oscillations in a Thermal Plasma, Phys. Rev. Lett. 61, 90 (1988).

[62] S. V. Bulanov, T. Zh. Esirkepov, M. Kando, J. K. Koga, T. Hosokai, A. G. Zhidkov, and R. Kodama, Nonlinear plasma wave in magnetized plasmas, Phys. Plasmas 20, 083113 (2013).

Correction: The article identification number was incorrect in Ref. [31] and has been fixed, enabling access to this source. 